\title{
Role of Strain Elastography in Assessment of Cervical Incompetence
}

\author{
Mohammed A. Salem $^{(1)}$, Ashraf T. Youssef ${ }^{(2)}$, Engy S. Elkayal ${ }^{(3)}$, Ahmed S. \\ Ragheb $^{(4)}$,Sara M.Abdelgaffar ${ }^{(5)}$. \\ (1) Professor of radiodiagnosis, Faculty of medicine ,Fayoum university \\ (2) Assistant professor of radiodiagnosis, Faculty of medicine ,Fayoum university \\ (3) Lecturer of radiodiagnosis, Faculty of medicine ,Fayoum university \\ (4) Professor of radiodiagnosis,Faculty of medicine, Zagazig University \\ (5) Resident of radiodiagnosis , ministry of health
}

Corresponding author:Dr. Sara Abdelgaffar

E-mail address: ziadwomar@gmail.com

Tel:01102523333

Fax: +2 084636583

\begin{abstract}
Background: Obstetricians exert a lot of efforts to decrease the rate of perinatal morbidity and mortality of mothers and their offspring. Preterm delivery is known to be one of the chief causes for this burden. The aim of this study was to estimate the potential value of elastographic evaluation of cervical canal stiffness at 12-14 weeks of pregnancy in women with cervical incompetence in the prediction of spontaneous preterm delivery.
\end{abstract}

Patient and Methods: This prospective observational study included 40 women presenting for the routine first trimester ultrasound scan between 12-14 weeks of pregnancy. Ultrasound examinations of the cervix were performed transvaginally (TV). The following data were recorded: elastographic color assessment of the cervical canal and ultrasound cervical length at 12-14 and weeks of pregnancy; maternal age; obstetrical history; gestational age at birth. Elastographic assessment of the cervical canal was performed using a color map: Red (soft), Yellow (medium soft), Green (medium hard) and Purple (hard). If two colors were visible in the region of the cervical canal, the softer option was noted.

The collected data were organized, tabulated and statistically analyzed using SPSS software statistical computer package version 18 (SPSS Inc., USA). For quantitative data, the mean, standard deviation (SD), and range were calculated. Mann-Whitney-U test and Kruskal- Wallis test were used as a test of significance to compare between two and three groups, respectively. Qualitative data were presented as number and percentages, chi square $(\chi 2)$ was used as a test of significance. Spearman correlation test was run to identify relation between cervical elasticity and cervical length. For interpretation of results of tests of significance, significance was adopted at $\mathrm{p}$ value $\leq 0.05$.

Results: The number of preterm deliveries ( < 37 weeks of pregnancy) was significantly higher in the Red and Yellow groups, than in the Green and Purple groups. The sensitivity, specificity, NPV and PPV in predicting preterm delivery for Red color group in patients with 
cervix length $<25 \mathrm{~mm}$ were $62.5 \%, 71.9 \%, 35.7 \%$ and $88.5 \%$ respectively with total accuracy of

$70 \%$. When adding the "yellow color" the sensitivity was raised to $87.5 \%$, yet, the

specificity dropped to $62.5 \%$ and the total accuracy dropped to $67.5 \%$.

Conclusion: Elastographic assessment of the cervical canal at 12-14 weeks of pregnancy may

\section{INTRODUCTION}

The cervix is continuous with the lower part of the uterus. Its proximal portion is located in the abdomen and its distal portion in the vagina. It has a narrow central canal which runs along its entire length, connecting the uterine cavity and the lumen of the vagina. The opening of this canal into the uterus is called the internal os and the opening into the vagina the external os. It is a firm, cylindrical structure [1]. The length of a normal adult non-pregnant cervix is approximately $30 \mathrm{~mm}$, with an antro- posterior diameter ranging between 20 and $25 \mathrm{~mm}$ and a transverse diameter of 25-30 mm, although considerable variations occur due to age, parity and stage of menstrual cycle [2]. It is the part of the uterus responsible for maintaining pregnancy till term. As long as the cervix remains long and firm and its internal orifice (os) is closed, it can withstand enlargement of the uterine contents and resultant growing pressure. During normal pregnancy, the uterine cervix undergoes physiological changes in the extracellular matrix in order to reduce stiffness, but increase its tensile proprieties. The cervix ripens prior to Delivery then effaces and dilates with contractions of the uterus [3]. Ripening of the cervix can be assessed using the Bishop score and ultrasonographically determined length of the cervical canal and internal os. Consistency is one of the cervical properties that change during the course of the maturation process. identify patients with high risk of preterm delivery in women with cervical incompetence.

\section{KEYWORDS: Elastography, Preterm delivery, Ultrasonography, Cervical canal assessment.}

Until recently, cervical consistency has been assessed only manually, but in 2007, the first report on elastographic imaging of the cervix during pregnancy has been published [4]. Cervical incompetence is an important contributor to pre-term birth and second trimester pregnancy loss. It is defined as the inability to support a full-term pregnancy because of a functional or structural defect of the cervix. It is reported that the rate of cervical incompetence is between $0.1 \%$ and $2 \%$, and is estimated to account for $15 \%$ of the recurrent pregnancy losses between 16 and 28 weeks. Identifying patients threatened with cervical incompetence and treating them remains one of the main obstetric challenges [5].

Ultrasound elastography (UE) depicts the stiffness of tissues. It was first described in the 1990s. It has been further developed and refined in recent years to enable quantitative assessments of tissue stiffness. Elastography methods take advantage of the changed elasticity of soft tissues resulting from specific pathological or physiological processes. Conventional ultrasound (US) has the advantage of being an inexpensive, versatile, and widely available modality that can be used at the bedside, which also applies to USE [6]. Strain elastography (SE) is available in most high-end ultrasound systems. SE measures axial displacement of tissue caused by mechanical 
stress in real-time. The stress is either applied externally with the transducer by the operator or by physiological shifts inside the patient.

Transducer stress is applied by continuously compressing and decompressing the region of interest, a few millimeters of axial transducer movement being sufficient [7].

The elastogram is derived from data of the change of radio frequency signals before and after compression. It is displayed in a splitscreen mode with the conventional B-mode image and the elastogram on the monitor. The elastogram may be displayed as a coloroverlay on the B-mode picture Elastographic assessment of the internal os will be performed using a color map: red (soft), yellow (medium soft), green (medium hard) and

purple (hard). If two colors were visible in the region of the internal os, the softer option was noted [8].

The clinical importance of assessing the uterine cervix during pregnancy is obvious. It is well known that the cervix undergoes biochemical changes in advance of morphological change during pregnancy. Thus, there is clearly a clinical need for cervical elastography in the evaluation of biochemical factors in combination with cervical length, which only assesses morphological changes [8].

\section{PATIENTS AND METHODS}

We conducted a descriptive cross sectional study in the ultrasound unit of radiology department - Fayoum University Hospital between October 2017 - October 2018 after obtaining Approval from research ethical committee in Fayoum University. 40 patients who were eligible for enrollment in this study (being referred from obstetrics and gynecology department, in addition to those who are accidentally discovered during ultrasound examination in Ultrasound Unit) were presented to assess the elasticity characteristics of the cervix and to evaluate the diagnostic performance of Strain Elastography (SE), with regards to cervical incompetence. The diagnosis of cervical incompetence was based on ultrasound measurement of the cervical canal length being less than $3 \mathrm{~cm}$. Inclusion criteria were patients referred to Radiology Department in Fayoum University Hospital for evaluation of cervical incompetence, patients who provided written consent, history of abortion or preterm labor, pregnant women between 12-14 weeks, single embryo and cervical length $<30 \mathrm{~mm}$. Exclusion criteria were declined consent, history of cervical surgery, Pregnant women after 14 weeks and Pregnant women who underwent cerculage operation. The following variables were to be assessed in this study; cervical length, internal os diameter, presence of cervical funneling and/or bulging of membranes or fetal parts into cervical canal. Operator was a single radiologist who was aware of the clinical information and suspected diagnosis in each patient. Baseline B-mode ultrasound of the cervix was performed first to identify cervical length.

The technique for SE was to image the cervix with minimal pressure induced by the transducer.

After a few seconds of immobilization to allow the SE image to stabilize, the image was frozen and saved. The area of interest was set to be the cervical canal, and it was displayed on a semitransparent color map of tissue stiffness overlaid on the gray scale image, ranging from red indicating the lowest stiffness, to purple indicating the highest stiffness. Strain elastography was carried out with the following color coding 
regarding cervical stiffness; Red (soft), yellow (medium soft), green (medium hard) and purple (hard) [Figure (1)]. If two colors were visible in the region of the internal os, the softer option was noted.

\section{STATISTICAL ANALYSIS}

The collected data were organized, tabulated and statistically analyzed using SPSS software statistical computer package version 18 (SPSS Inc., USA). For quantitative data, the mean, standard deviation (SD), and range were calculated. Mann-Whitney-U test and Kruskal- Wallis test were used as a test of significance to compare between two and three groups, respectively. Qualitative data were presented as number and percentages, chi square $(\chi 2)$ was used as a test of significance. Spearman correlation test was run to identify relation between cervical elasticity and cervical length. For interpretation of results of tests of significance, significance was adopted at $p$ value $\leq 0.05$

\section{RESULTS}

Our study was conducted in Radiology department, Fayoum University hospital, including

40 Pregnant women with single embryo at 12-14 weeks gestation in whom cervical

length $<30 \mathrm{~mm}$ with history of previous abortion or preterm labor. Their age ranged between

19 and 34 years $(25.5 \pm 3.9)$, the cervical length was identified among them to be less than 30 $\mathrm{mm}$, ranging between (23-28.2) with mean length of $(26.4 \pm 1.3)$, their gravidity ranged between (1-5) offsprings with mean $(2.5 \pm 0.9)$ and their parity ranged between (0-4) with mean $(1.1 \pm 0.9)$. (Table [1])
Pregnancy outcome: Data about the delivery were obtained from all the patients. Mean gestational age was $37.7 \pm$ 4.1 weeks ranging from 14 to 40 weeks. Out of 40 patients, 9 patients $(22.5 \%)$ had preterm deliveries (before 37 week) and 31 patients $(77.5 \%)$ had full term deliveries (after 37 weeks).

Cervical elastography data: At $12-14$ weeks of pregnancy, the cervical canal was evaluated by strain elastography by color mapping, and assessed as (according to a four-step color scale) as purple (hard), green (medium hard), yellow (medium soft) and red (soft). Our study showed that 14 patients had red cervical canal (soft) (35\%), 5 patients had yellow cervical canal (medium soft) $(12.5 \%)$, 17 patients had green cervical canal (medium hard) $(42.5 \%)$ and 4 patients had purple cervical canal (hard) $(10 \%)$.

\section{Cervical canal length and elastography:}

Cervical canal length was measured in each elastography color group as follows: in the Red group (Soft cervix) mean cervical canal length was $26.2 \pm 1.6 \mathrm{~mm}$, in the Yellow group (Medium Soft cervix) it was $26.3 \pm 1.7 \mathrm{~mm}$, in the Green group (Medium hard cervix) it was $26.4 \pm 0.9$, while in the Purple group (hard cervix) mean cervical canal length was $27.6 \pm$ $0.5 \mathrm{~mm}$. In the warm color group (red and yellow) mean cervical length was $26.2 \pm 1.9 \mathrm{~mm}$ while in the cold color group (green and purple) it was $26.9 \pm 0.7 \mathrm{~mm}$. There was no statistically significant difference in mean cervical canal length neither among the four color groups ( $\mathrm{p}$ $=0.160$ ) nor between the warm colors (red and yellow) and the cold colors (green and purple) groups $(\mathrm{p}=0.14)$.

\section{Cervical elastography and Pregnancy} outcome: The numbers and percentages of preterm deliveries in respective groups were: Red (3 out of 14 patients, 21.4\%), 
Yellow (4 out of 6 patients, $66.7 \%$ ), Green (1 out of 16 patients, $6.25 \%$ ) and Purple (1 out of 4 patients, 25\%)(Table [2]). Although there was no significant difference in mean cervical canal length neither among the four color groups nor between the warm colors and the cold colors groups, The number of preterm deliveries was significantly higher $[p=0.032]$ in the warm group (red and yellow colors) [preterm delivery occurred in 7 out of 20 patients $(35 \%)$ ] than in the cold group (purple and green colors) [preterm delivery occurred in 2 out of 20 patients (10\%)]. However, there was no statistically significant difference in the number of preterm deliveries neither between the red and yellow groups $[\mathrm{p}=0.054]$ nor between the green and the purple groups $[\mathrm{p}=0.089]$.

\section{Diagnostic accuracy of cervical incompetence} in predicting preterm labour: It was found that the Red color group (soft cervix in elastography reading) has $62.5 \%$ sensitivity and $70.9 \%$ specificity, with a total diagnostic accuracy of $70 \%$ in predicting preterm delivery. When adding the "yellow color" the sensitivity was raised to $87.5 \%$, the specificity to $72.5 \%$ and the total accuracy increased to $74.3 \%$ of cases of preterm delivery. The sensitivity, specificity, negative predictive value (NPV), positive predictive value (PPV) at $95 \%$ confidence intervals for various categories of cervical canal elastography assessment for prediction of high risk of preterm delivery patients are shown in table [3].

\section{DISCUSSION}

It is known that the properties of cervical tissue change during pregnancy and labor. Mechanical devices have been used to objectively assess cervical mechanical properties in pregnancy: it has been shown that the cervix becomes softer and more distensible as the pregnancy progresses [9]. At present screening for patients with high risk of preterm birth is based mainly on the measurement of cervical length at the first trimester scan. Out of all cases of preterm deliveries ( $<35$ weeks) in $62.7 \%$ the cervix length was above $25 \mathrm{~mm}$. These findings justify the search for additional diagnostic tests in patients with normal cervix length. Even in patients with a normal cervix length at 12-14 weeks scan it is possible to identify a group of patients with high risk of preterm birth. It has to be stressed that all of these patients according to current guidelines were classified as lowrisk of preterm birth and did not qualify for additional interventions and monitoring [10]. Cervical tissue strain assessed by elastography was found to be more strongly associated with cervical length than with gestational age at examination [11].

In our study we used strain elastography to assess the stiffness of cervical canal using a four step color scale. We conducted a study that included 40 patients identified to have short cervix $(<30 \mathrm{~mm})$ to evaluate the role of strain elastography in predicting preterm delivery. Two categories were used to describe cervical canal elastographic readings:

The warm group (softer cervical canal): red (soft) and yellow (medium soft)

The cold group (harder cervical canal): green (medium hard) and purple (hard).

Our results demonstrated that although there was no significant difference in cervical canal length, yet cervical elastography had significant diagnostic accuracy in predicting the risk of preterm birth during the second trimester of pregnancy. For patients with red scale (soft cervical canal), sensitivity of predicting preterm labour was $62.5 \%$ and specificity was $70.9 \%$ with a total diagnostic accuracy of $71 \%$ of cases. When we combined red and yellow cervical canal elastographic readings (i.e. the warm color group), we have observed an increase in sensitivity 
(87.5\%), specificity (72.5\%) and total diagnostic accuracy $(74.3 \%)$.

Wozniak et al conducted a prospective observational study on 333 low-risk, asymptomatic women presenting for the routine second trimester ultrasound scan. The following data were recorded: elastographic color assessment of the internal os and ultrasound cervical length at 18-22 and 30 weeks of pregnancy; maternal age; obstetrical history; presence of cervical funneling at 30 weeks of pregnancy; gestational age at birth.

Elastographic assessment of the internal os was performed using a color map: red (soft), yellow (medium soft), green (medium hard) and purple (hard). The number of preterm deliveries (<37 weeks of pregnancy) was significantly higher in the red and yellow groups, than in the blue and purple groups. The sensivity and specifity for both red and yellow internal os assessment in predicting preterm delivery were $85.7 \%$ and $97.6 \%$ respectively. They concluded that Elastographic assessment of the internal cervical os at 18-22 weeks of pregnancy may identify patients with high risk of preterm delivery in low-risk, asymptomatic women [8]. This is in agreement with the results of current study.

In another study by Wozniak et al, 109 patients with cervical length of $\leq 25 \mathrm{~mm}$ at $18-22$ weeks were enrolled. Stiffness of the internal cervical os was assessed by elastography. Elastographic assessment of the internal os was performed using a color map: red (soft), yellow (medium soft), blue (medium hard), and purple (hard). 45 cases of PreTerm Deliveries (PTDs) ( $<37$ weeks of pregnancy) were found in the studied population. The number of PTDs was significantly higher in the red group, than in the blue and purple groups. The sensitivity, specificity, NPV and PPV for the assessment of both, red and yellow internal os for predicting preterm delivery were
$82.2 \%, 75.0 \%, 84.0 \%$ and $72.5 \%$ respectively. They suggested inclusion of both, red and yellow

(warm) colors as cut-off value for elastography suggested as predictors of PTD. Also they concluded that elastographic evaluation of the internal cervical os at 18-22 weeks of pregnancy in patients with short cervical length may be useful in predicting PTD [12]. The postulated results are in agreement with the results of current study.

Hernandez-Andrade and colleagues enrolled189 women at 16-24 weeks of gestation for evaluation by Ultrasound elastography to estimate cervical strain in three anatomical planes: one mid-sagittal in the same plane used for cervical length measurement and two cross sectional images: one at the level of the internal cervical os, and the other at the level of the external cervical os. In each plane, two regions of interest (endocervix and entire cervix) were examined. Strain measurements obtained in a cross sectional view of the internal cervical os were significantly associated with Spontaneous PreTerm Delivery (sPTD). Women with strain values $\leq 25$ th centile in the endocervical canal (0.19) and in the entire cervix (0.14) were $80 \%$ less likely to have a sPTD than women with strain values $>25$ th centile. They concluded that increased strain levels (increased cervical softness determined by cervical strain elastography) are associated with higher risk of preterm delivery [13] and their results are in agreement with results of current study.

At present various groups propose different standardization approaches. For instance, Fruscalzo compressed the cervix to obtain maximal compression of the anterior lip, until the posterior lip was displaced [14]. While Swiatkowska-Freund generated elastographic images by patient's breathing and arterial pulsation without any additional force 
applied [15]. Molina advanced the probe into cervical tissue by about $1 \mathrm{~cm} \mathrm{[16].} \mathrm{We} \mathrm{have}$ used the last of these techniques adopted by Molina et al - it has to be stressed that since no reliability studies of SwiatkowskaFreund's method were performed, it seems of utmost importance to investigate the operatorindependence and the reproducibility of this technique [17].

In summary, elastography allows the assessment of cervical canal stiffness in pregnancy. Secondly, elastographic abnormalities may precede and predict clinical and ultrasound findings (cervical shortening and funneling). Thus elastographic assessment of the internal cervical os may provide additional information in preterm delivery risk assessment.

\section{LIMITATIONS}

Our study has some limitations. First of all, elastography lacks standardization. That is why to avoid the possible inter observer bias, only one experienced examiner performed all examinations. However, our study is an initial observation, showing that by using elastography it is possible to identify patients threatened with preterm delivery. Further studies focusing mainly on the standardization and intra- and inter-observer variability of this technique are needed. In addition, some patients refused to participate in the study

\section{CONFLICT OF INTEREST}

None of the authors declared any conflicts of interest. while others failed to complete the study and were not available to be contacted to obtain pregnancy outcome data. They were not included in study population. Furthermore, some patients underwent prophylactic cerculage operations in second trimester so they were excluded from the study population as pregnancy outcome data will not be correlated with cervical elastographic data obtained.

\section{CONCLUSION AND RECOMMENDATIONS}

In conclusion, elastography was found to be of high sensitivity and specificity in detecting cervical incompetence. Elastographic abnormalities may precede ultrasound and clinical findings, such as cervical shortening and funneling. It is simple, fast and non-invasive technique that adds more to the total accuracy of the conventional first trimester US exam increasing the confidence of the diagnosis of cervical incompetence. It is simple, fast and non-invasive technique that adds more to the total accuracy of the conventional first trimester US exam increasing the confidence of the diagnosis. It is our recommendation to consider cervical elastography as a part of routine cervix ultrasound exam using color coding to increase confidence of diagnosis. Further studies are needed to consolidate the evidence of such practice. 
ISSN: 2536-9474 (Print)

ISSN: 2536-9482 (Online)
Fayoum University Medical Journal

Original article / FYMJ

Abdelgaffar et al., 2018, 1 (2), 9-18

Table[1] basic characteristics of study participants $(\mathrm{N}=40)$

\begin{tabular}{lll} 
Variable & Mean \pm SD & Range \\
\hline Maternal age (years) & $25.5 \pm 3.9$ & $19-34$ \\
Gravidity & $2.5 \pm 0.9$ & $1-5$ \\
Parity & $1.1 \pm 0.9$ & $0-4$ \\
Cervical length $(\mathrm{mm})$ & $26.4 \pm 1.3$ & $23-28.2$ \\
\hline
\end{tabular}

Table[2] pregnancy outcome in relation to cervical elastography

\begin{tabular}{|c|c|c|c|c|c|c|}
\hline \multicolumn{2}{|c|}{ Variable } & $\begin{array}{l}\text { Preterm } \\
(\mathrm{N}=9)\end{array}$ & $\begin{array}{l}\text { Full.term } \\
\qquad N=31)\end{array}$ & n & \multicolumn{2}{|c|}{ P.value } \\
\hline \multirow{2}{*}{ Wamn group } & Sott (red) & $3(21.4 \%)$ & $11(78.6 \%)$ & 14 & \multirow{2}{*}{0.054} & \multirow{4}{*}{0.032} \\
\hline & Medium sot (yellow) & $4(66.7 \%)$ & $2(33.3 \%)$ & 6 & & \\
\hline \multirow{2}{*}{ Cold group } & Medium hard (green) & $1(6.25 \%)$ & $15(93,75 \%)$ & 16 & \multirow{2}{*}{0,089} & \\
\hline & Hard (purple) & $1(25.0 \%)$ & $3(75.0 \%)$ & 4 & & \\
\hline \multicolumn{2}{|r|}{ n } & 9 & 31 & 40 & & \\
\hline
\end{tabular}

Table[3] diagnostic accuracy of cervical elasticity in predicting preterm labour

\begin{tabular}{|c|ccccc|}
\hline $\begin{array}{c}\text { Cervical Elasticity } \\
\text { Category }\end{array}$ & $\begin{array}{c}\text { Sensitivity } \\
(\%)\end{array}$ & $\begin{array}{c}\text { Specificity } \\
(\%)\end{array}$ & $\begin{array}{c}\text { PPV } \\
(\%)\end{array}$ & $\begin{array}{c}\text { NPV } \\
(\%)\end{array}$ & $\begin{array}{c}\text { Total accuracy } \\
(\%)\end{array}$ \\
\hline Red & 62.5 & 70.9 & 70.7 & 88.5 & 71.0 \\
\hline Red + Yellow & 87.5 & 72.5 & 77.3 & 95.2 & 74.3 \\
\hline
\end{tabular}

Figure 1: Elastographic images of cervical canal at 12-14 weeksof gestation .cervical canal stiffness was assessed as:soft consistancy (red) [A] and hard cosistancy (purple) [B]

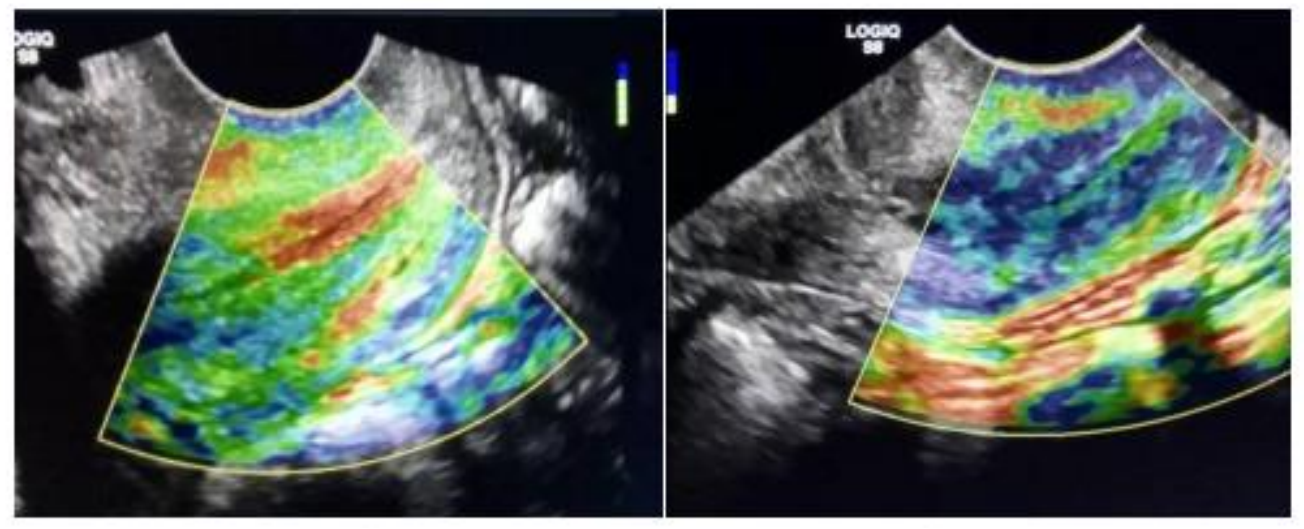




\section{REFERENCES}

[1] Myers KM, Feltovich H, Mazza E, Vink J, Bajka M, Wapner RJ et al (2015): The mechanical role of the cervix in pregnancy. Journal of Biomechanics, 48(9), 1511-1523

[2] Nott JP, Bonney EA, Pickering JD and Simpson NA (2016): The structure and function of the cervix during pregnancy. Translational Research in Anatomy, 2, 1-7

[3] Kim H and Hwang HS (2017): Elastographic measurement of the cervix during pregnancy: Current status and future challenges. Obstetrics and Gynecology Science, 60(1), 1-7

[4] Swiatkowska-Freund $M$ and Preis K (2011): Elastography of the uterine cervix: implications for success of induction of labor. Ultrasound Obstet Gynecol, 38: 52-56

[5] Zhu L, Chen H, Chen L, Liu Y, Tan J, Wang Y et al (2015): Effects of Emergency Cervical Cerclage onPregnancy Outcome: A Retrospective Study of 158 Cases . Med Sci Monit, 21: 1395-1401

[6] Rosa MS, Liau J, El Kaffas A, Chammas MC and Willmann JK (2017): Ultrasound Elastography: Review of Techniques and Clinical Applications. Theranostics, 7(5): 1303-1329

[7] Carlsen JF, Ewertsen C, Lönn L and Nielsen MB (2013): Strain Elastography Ultrasound:AnOverview with Emphasis on Breast Cancer Diagnosis. Diagnostics (Basel), 3(1): 117-125

[8] Wozniak S, Czuczwar P, Szkodziak P, Milart P, Wozniakowska $E$ and Paszkowski T (2014): Elastography in predicting preterm delivery in asymptomatic, low-risk women: a prospective observational study. $B M C$ Pregnancy Childbirth, 14:238
[9] Bauer M, Mazza E, Jabareen M, Sultan L, Bajka M, Lang U et al (2009): Assessment of the in vivo biomechanical properties of the human uterine cervix in pregnancy using the aspiration test: a feasibility study. Eur J Obstet Gynecol Reprod Biol, 144(1):S77-S81

[10] O'Hara S, Zelesco M, and Sun Z (2013): Cervical length for predicting preterm birth and a comparison of ultrasonic measurement techniques. Australasian Journal of Ultrasound in Medicine,16(3), 124-134

[11] Hernandez-Andrade E, Hassan SS, Ahn H, Korzeniewski J, Yeo L, Chaiworapongsa TE et al (2013): Evaluation of cervical stiffness during pregnancy using semiquantitative ultrasound elastography. Ultrasound Obstet Gynecol, 41: 152-161

[10] House M, Kaplan DL and Socrate S (2009): Relationships between mechanical properties and extracellular matrix constituents of the cervical stroma during pregnancy. Semin Perinatol, 33: 300-307.

[12] Wozniak S, Czuczwar P, Szkodziak P, Wrona W and Paszkowski T (2015): Elastography for predicting preterm delivery in patients with short cervical length at 18-22 weeks of gestation: a prospective observational study. Ginekol Pol, 86(6), 442-447

[13] Hernandez-Andrade E, Romero R, Korzeniewski SJ, Ahn H, AuriolesGaribay A, Garcia $M$ et al (2014): Cervical strain determined by ultrasound elastography and its association with spontaneous preterm delivery. Journal of Perinatal Medicine, 42(2), 159-169 
[14] Fruscalzo A, Steinhard J, Londero AP, Fröhlich C, Bijnens B, Klockenbusch W et al (2013):

Reliability of quantitative elastography of the uterine cervix in at-term pregnancies. $J$ Perinat Med, 41:421-427

[15] Swiatkowska-Freund $M$ and Preis $K$ (2011): Elastography of the uterine cervix: implications for success of induction of labor. Ultrasound Obstet Gynecol, 38: 52-56
[16] Molina FS, Gómez LF, Florido J, Padilla MC and Nicolaides KH (2012): Quantification of cervical elastography: a reproducibility study. Ultrasound Obstet Gynecol, 39:685-689

[17] Yamaguchi S, Kamei Y, Kozuma S, Kawashima H, Komura $M$ and Sugiyama M (2007): Tissue elastography imaging of the uterine cervix during pregnancy. J Med Ultrasonics, 39: 209-210 